\title{
Psychoanalyse auf Arabisch
}

Katrin Hartmann (Bern)

Zusammenfassung: Der vorliegende soziologische Artikel thematisiert, wie in den letzten Jahrzehnten arabische PsychoanalytikerInnen und Gruppierungen die Etablierung und Professionalisierung der Psychoanalyse in einzelnen Ländern der arabischen Welt vorantreiben. Er gibt Einblicke in die sich dabei abspielenden heterogenen Geschichten der arabischen psychoanalytischen Bewegungen. Im Zentrum stehen die psychoanalytischen Communities Syriens, Ägyptens und des Libanon. Die Autorin streift ausserdem Fragen der Arabisierung der Psychoanalyse am Beispiel der Übersetzbarkeit der psychoanalytischen Literatur in die arabische Sprache. Ebenso umreisst sie verschiedene Lesarten dessen, wie der Aneignungsprozess der Psychoanalyse in der arabischen Welt verstanden werden kann.

Schlüsselwörter: Psychoanalyse, arabische Welt, Professionalisierung, Institutionalisierung, Islam

Welcher Psychoanalytikerin ist es in den letzten Jahren widerfahren, dass man ihr auf Youtube mit einem Song huldigte? Für welchen Psychoanalytiker wurde je ein Flashmob gestartet? Diese Solidaritätsbekundungen galten im Herbst 2011 der syrischen Psychoanalytikerin Rafah Nached, die wegen ihrer angeblich systemkritischen, eher jedoch psychoanalytischen Diskussionsabende von der Regierung Assads am Flughafen in Damaskus in Haft genommen wurde. Sie war auf dem Weg nach Frankreich gewesen, um bei der Geburt ihres Enkelkinds dabei zu sein. Der Flashmob wurde im Rahmen einer Tagung der Ecole de la cause freudienne in Szene gesetzt. 2000 PsychoanalytikerInnen unterzeichneten dabei eine Motion, mit der die Freilassung von Nached gefordert wurde. «Libérez Rafah », so heisst der französische Youtube-Song. Die Aktion war erfolgreich. Nach 67 Tagen wurde die 66-jährige Psychoanalytikerin aus der Haft entlassen (Savigneau, 2011 und Bruno, 2012).

Dieses Beispiel dient dreierlei Zwecken. Zum einen steht es sinnbildlich dafür, dass die Psychoanalyse in der arabischen Welt angekommen ist: Es gibt im Machrek und im Maghreb psychoanalytische Tätigkeiten. Zweitens illustriert es, 
dass das psychoanalytische Denken immer wieder autoritären Regierungskreisen in den arabischen Ländern ein Dorn im Auge war beziehungsweise ist. Drittens steht das Beispiel für die typische Vernetzung zwischen den ProtagonistInnen der arabischen Psychoanalyse mit denen des Westens: eine in Syrien arbeitende Psychoanalytikerin, für die in Paris KollegInnen der psychoanalytischen Bewegung auf die Strasse gehen.

Im nachfolgenden Artikel stelle ich drei typische VertreterInnen der Psychoanalyse der arabischen Welt vor: neben Rafah Nached noch einen weiteren Psychoanalytiker, den Libanesen Adnan Houbballah. Weiter gebe ich Einblicke in das Leben eines arabischen Intellektuellen, der sich über Jahrzehnte hinweg mit der Psychoanalyse auseinandergesetzt hat: der Syrer George Tarabishi. Neben der Beschreibung dieser Persönlichkeiten skizziere ich die Entwicklung der psychoanalytischen Gesellschaften, die heute in der arabischen Welt eine Rolle spielen. Dabei weise ich auf Fragen hin, die mit der arabischen Übersetzung des psychoanalytischen Wissensgebäudes verbunden sind. Den Abschluss des Artikels bildet ein Blick auf die Psychoanalyse in Ägypten. Methodisch baut der Artikel auf den Erkenntnissen auf, die ich während einer Feldforschung über die Psychoanalyse im Libanon gewinnen konnte (Hartmann, 2007). Dieses Vorwissen half mir, diesen Artikel zu schreiben, konkret stütze ich mich jedoch mehrheitlich auf neuere englisch- und französischsprachige Literatur.

Für eine bessere Orientierung durch den Artikel sei vorangeschickt, dass ich darin zwischen verschiedenen Ländern, Zeiten und Personen hin- und herspringe. Als Rahmenstruktur dieser arabischen «Studienreise» stütze ich mich auf ein konzeptuelles Gerüst, das von Schülein (2007), der die Institution «Psychoanalyse» seit vielen Jahren erforscht, konzipiert wurde. Es handelt sich um ein Drei-PhasenModell, durch das der Prozess der lokalen Etablierung der Psychoanalyse beschrieben und eingeordnet werden kann.

Nached ist eine typische Protagonistin für die, wie Schülein (2007, S. 259) es formuliert, Pionierphase der Etablierung der Psychoanalyse in einem noch relativ unberührten Kontext. Als ich im Jahr 2000 meine Feldforschung im Libanon durchführte, hörte ich, dass im Nachbarland Syrien mit seiner rund 16 Millionen zählenden Einwohnerschaft drei weitere KollegInnen psychoanalytisch arbeiten. Diese Vorstellung beeindruckte mich: südlich vom Libanon Israel, mit seiner etablierten Psychoanalyse-Community; östlich davon Syrien, in dem hartnäckige EinzelkämpferInnen tätig waren, während im winzigen Libanon eine kleine couragierte psychoanalytische Bewegung existierte, die sich für die Etablierung der Psychoanalyse seit mehr als 20 Jahren stark machte. 
Diese drei Länder können exemplarisch als Idealtypen für die drei Stadien der Psychoanalyse-Etablierung aufgefasst werden, welche Schülein (2007) in seinem Werk über das Gesellschaftsbild der freudschen Psychoanalyse ausarbeitete: die Pionierphase, die Phase der Konsolidierung und Expansion und schliesslich die letzte Phase, der Beginn eines Prozesses der Normalisierung (ebd., S. 260).

Schülein zufolge (ebd., S. 259f.) geht es in der Pionierphase darum, für ein noch unentwickeltes Thema inhaltlich Profil und Terrain zu erarbeiten und sozial abzusichern. Natürlich kann der Psychoanalyse nicht nachgesagt werden, dass sie ein inhaltlich unentwickeltes Thema sei. Im arabischen Kontext mit seinen quantitativ wenigen psychoanalytisch Tätigen ist die Rezeption und Etablierung der Psychoanalyse aus einer sozialen Perspektive dennoch als rudimentär zu bezeichnen. Der Psychoanalyse als Wissenschaft, Sozialorganisation und Berufsstand fehlt es in diesem Kontext in vieler Hinsicht an klaren Konturen. Für die Pioniere der ersten Phase wichtig sind Eigenschaften, die diese befähigen - trotz Widerstand von Institutionen und Gruppen -, gegen den «Sog der herrschenden Normalität eine abweichende Linie zu verfolgen», auch wenn diese Innovation «noch nicht als präzise Alternative definiert ist» (ebd., S. 259). Die Pioniersituation zeichnet sich daher thematisch wie sozial durch «unentwegtes Improvisieren» aus. Die «Fähigkeit zu skrupellosem Ausprobieren, zu schnellen Umstellungen und zum Aushalten von Disparitäten» macht so gesehen den Sozialtyp des Pioniers bzw. der Pionierin aus (ebd., S. 260). Viele dieser beschriebenen Züge lassen sich bei Nached ausmachen.

\section{Rafah Nached}

Nached wurde 1945 in Aleppo geboren; sie stammt aus einer sunnitischen Familie. In Beirut studierte sie an der Université Saint-Joseph Philosophie. Sie setzte ihre Studien in Paris fort, bildete sich in klinischer Psychologie aus und wurde schliesslich Psychoanalytikerin lacanscher Richtung. Mit 40 Jahren begann sie, in Syrien psychoanalytisch zu arbeiten. Sie war damals die erste und einzige Psychoanalytikerin. Erste Station war ein hospice de vieillards in Aleppo, in dem Menschen aller Altersgruppen mit verschiedensten psychischen Störungen behandelt wurden. Danach folgte die Tätigkeit in einem Zentrum für geistig Behinderte. Parallel dazu arbeitete sie in eigener Praxis in Damaskus. In ihrem Umfeld sah sich Nached grossen Widerständen ausgesetzt: von der universitären Welt, welche die Psychoanalyse als obsolet und unnütz verurteilte, durch die Psychiatrie, die Kranke allein mit Medikamenten und Elektroschocktherapie behandelte, durch die Gesellschaft, die Gott als Lösung für alles anrief und das magische Denken als Heilungsmittel erster Wahl betrachtete (Nached, 2010, S. 18f.). «A cause de tous 
ces éléments, j'ai dû parler de la psychanalyse et l'exercer dans la discrétion la plus totale» (ebd., S. 20).

Mit einem holländischen jesuitischen Analytiker begann sie, im Maison des jésuites regelmässige Vorlesungen zur Psychoanalyse abzuhalten. Die psychoanalytische Idee verbreitete sich, auch wenn der Kontrast zwischen der wachsenden Nachfrage in ihrer privaten Praxis und dem Widerstand aller grösseren Institutionen nach wie vor bestehen blieb (ebd., S. 20).

Ein dritter (damals verantwortlicher) Psychoanalytiker beendete diese öffentlichen Foren aus der Haltung heraus, dass es zu ungewiss sei, wohin die Vorlesungsreihe führen könnte. Nached war wieder auf sich allein gestellt. Sie näherte sich daraufhin der psychoanalytischen Bewegung des Libanon an und organisierte auf eigene Faust Seminare, zu denen sie libanesische PsychoanalytikerInnen lud.

Im Jahr 2000 gründete sie die Ecole psychanalytique de Damas. Anstoss dazu hatte ein aus England zurückgekehrter Psychiater gegeben, der Weiterbildungen für MedizinerInnen anbieten wollte. Diesen Seminaren in einem kleinen Spital in einem Damaszener Vorort schlossen sich PsychologInnen der Universität an. Um den Interessierten eine fundierte Ausbildung zu ermöglichen, wandte sich Nached an KollegInnen der französischen Psychoanalysebewegung, insbesondere an diejenigen unter ihnen, die auf die Vermittlung der Psychoanalyse spezialisiert waren. Während neun Jahren wurden regelmässig Seminare abgehalten, meistens in französischer Sprache mit einer arabischen Simultanübersetzung (ebd., S. 123).

Nachdem der syrische Volksaufstand begonnen hatte, organisierte Nached ab Frühjahr 2011 offene Workshops gegen die sich ausbreitende Angst innerhalb der Bevölkerung. Gemeinsam mit dem Jesuitenpater und Psychoanalytiker Rami Elias hielt sie diese in wöchentlichem Rhythmus für Menschen aller Konfessionen und politischen Richtungen ab. Sechs Monate später kam es zu Nacheds Verhaftung. Vorgehalten wurde ihr, zur Destabilisierung des Staates beigetragen zu haben. Die psychoanalytische Bewegung Frankreichs begann sich kurz nach ihrer Inhaftierung zu mobilisieren und gelangte ans europäische Parlament, von dem es forderte, sich für die Freilassung von Nached einzusetzen. Das grosse Ausmass der Mobilisierung für die syrische Psychoanalytikerin lässt sich wohl so erklären, dass der Fall Nached, wie es Julia Kristeva formuliert, zu einem historischen Sinnbild «pour la psychanalyse, pour la politique, pour les femmes » wurde (zit. nach: Savigneau, 2011).

\section{Georges Tarabishi}

Die Züge eines eigenwilligen Nonkonformisten zeigt auch Tarabishi, der in den 1970er-Jahren einen Beitrag für die spätere Expansion der Psychoanalyse in 
der gesamten arabofonen Region leistete. Tarabishi ist ein syrischer Intellektueller, der als zentrale Figur betrachtet wird hinsichtlich der frühen Übersetzungen von Freuds Werken ins Arabische.

Der 1939 geborene Tarabishi, ursprünglich ein Lehrer, wandte sich früh dem Journalismus zu. Zwischen 1973 und 1987 übersetzte er eine Vielzahl der Arbeiten von Freud ins Arabische - insgesamt waren es 33 Werke (Ben Slama, 2009, S. 9). Ungefähr parallel dazu begannen im Libanon die ersten AnalytikerInnen zu praktizieren. Tarabishi startete diese Arbeit in einer Zeit, in der im gesamten arabischen Raum noch keine psychoanalytische Gesellschaft existierte und die psychoanalytische Ausbildung über Ausbildungsexkursionen nach Europa erfolgte.

Ein Referenzrahmen oder eine klare Terminologie der arabischen psychoanalytischen Literatur existierten damals nicht, Freud-Übersetzungen kamen vorher vereinzelt aus ägyptischer Feder. Die 1958 in Kairo erschienene Übersetzung von L'intérpretation des rêves des bei Lacan ausgebildeten Psychoanalytikers Moustapha Safouan gilt bis heute als Referenzwerk für Arabischsprechende, die sich für die Psychoanalyse interessieren (Ben Slama, 2010, S. 84).

Tarabishi hatte sehr früh, ohne ausgewiesene Vorbildung, seine Übersetzungstätigkeit mit marxistischen und später existenzialistischen Texten begonnen. Er war ein grosser Verehrer Sartres, bis dieser im Sechstagekrieg eine proisraelische Position einnahm. Die Aura Sartres erlosch in der arabischen Welt innerhalb weniger Tage. Es war der Fall eines symbolischen Vaters. Mit der brennenden Frage, weshalb er absolutes Vertrauen in eine Vaterfigur gesetzt hatte, die sich vom einen Moment zum anderen in Schall und Rauch auflösen konnte, machte sich Tarabishi an Freuds Werk: Er wollte verstehen. Für Tarabishi war die Entdeckung Freuds wie der Schatz, den Ali Baba in der Höhle der 40 Räuber entdeckte - eine mit Reichtümern gefüllte Höhle, die ihm eine neue Welt erschloss (Zoueïn \& de Rochegonde, 2004, S. 2).

Tarabishis Übersetzungstätigkeit führte zu einer konsquenten Kursänderung in seinem Leben, sie war gespiesen durch die Erkenntnis: «Ce n'est pas nous qui éduquons nos enfants, ce sont eux qui nous font père... J’ai été enseigné par elle [die Psychoanalyse] » (Zoueïn \& de Rochegonde, 2004, S. 3). Tarabishi traf in dieser Zeit keine/n AnalytikerIn, obwohl er versucht war, dies zu arrangieren. Als christlicher progressiver Syrier hatte er Syrien verlassen, um sich im kosmopolitischen Beirut niederzulassen. Das Freud-Studium bewahrte ihn während des durch den libanesischen Bürgerkrieg erzwungenen Schweigens vor dem Gefühl des totalen Eingeschlossenseins und vor dem Verrücktwerden. Ein schützenderVater, der ihn gegen die Barbarei des Bürgerkriegs abschottete und der im Gegensatz zu Sartre 
auch nicht mehr «fiel», trotz Entdeckens von Freuds Mankos. Tarabishi wurde kein Psychoanalytiker, er benutzte jedoch die psychoanalytische Methode, um aus deren Perspektive Literaturkritik zu betreiben. L'oedipe dans le roman arabe hiess sein erstes Werk (Zoueïn \& de Rochegonde, 2004, S. 3).

Einschneidend für seine weitere berufliche Tätigkeit war die Auseinandersetzung mit dem Werk von Hanafi, einem der Meister der arabischen Moderne. In seinem Aufsatz Une psychanalyse de l'idéologie dans la culture arabe kritisierte Tarabashi dessen ideologische Rückbesinnung auf ein arabisch-islamisches Erbe als einen neurotischen Diskurs, mit dem die zwei historischen Niederlagen - die Kolonialisierung durch Frankreich und England und der Sieg Israels - durch die Schaffung einer «Renaissance de la patrimoine» verarbeitet wurden. In jüngster Zeit hat sich Tarabishi mit Veröffentlichungen gegen den Islamismus einen Namen gemacht (ebd., S. 4). ${ }^{1}$

Der Psychoanalyse wurde während Tarabishis Übersetzungstätigkeit sogar im weltoffenen Beirut mit Zwiespalt begegnet. Seine Übersetzungen wurden stellenweise zensuriert. So riss Tarabishis Verleger in Beirut, der dessen Übersetzung von Moïse et le monothéisme herausgab, den Passus heraus, in dem der Islam als Religion bezeichnet wurde, die von der jüdischen Religion abstamme. Tarabishi fügte für die zweite Auflage die fehlende Seite unbemerkt wieder ein. Erst bei der siebten Neuauflage wurde die Stelle erneut zensuriert (ebd., S. 5).

Als Schwierigkeit für die Übersetzungstätigkeit bezeichnet Tarabishi Freuds Schreibweise. Es war Tarabishi daran gelegen, eine poetische Form zu finden für die Art von Intimität, die er von Freud zu hören glaubte, auch wenn er von der französischen Fassung ausging. Als weitere Herausforderung musste er Kunstworte schaffen, da es Ausdrücke wie beispielsweise Identifikation im Arabischen nicht gibt. Eine zusätzliche Krux waren die in der Psychoanalyse enthaltenen Homonyme, die zwar eher bei Lacan eine Rolle spielen (zum Beispiel merund mère), aber dennoch auch bei Freud zu finden sind (ebd., S. 6).

\section{Eine arabische Sprache der Psychoanalyse}

Die Frage der Übersetzung der psychoanalytischen Literatur ins Arabische ist insofern wichtig, da diese den Weg vorbereitet, um die Psychoanalyse zu konsolidieren. Denn die Übersetzung des psychoanalytischen Theoriekorpus stellt eine Form der Aneignung in die eigene Sicht des Menschseins und der Welt dar, die immer auch durch die Sprache geprägt wird. Durch eine umfassende Übersetzung der psychoanalytischen Werke ins Arabische wäre ein wichtiger Beitrag geleistet, 
breitere Gesellschaftskreise zu erreichen und es der lokalen psychoanalytischen Community einfacher zu machen, sich untereinander zu verständigen.

Dass hier ein Nachholbedarf besteht, macht die Psychoanalytikerin Raja Ben Slama in einem Artikel deutlich (2009). Als sie im Jahr 2005 nach Kairo übersiedelte und nach psychoanalytisch interessierten KollegInnen Ausschau hielt, merkte sie, wie schwierig es war, sich in Gesprächen und Diskussionen untereinander zu verständigen (ebd., S. 1).

Grundsätzlich ist die arabische Sprache als semitische Nachbarsprache der europäischen Sprachen für die Übersetzung der freudschen Terminologie nicht eine derart grosse Herausforderung wie beispielsweise das Chinesische. Die Binarität des Psychischen und des Körperlichen existiert auch in der arabischen Sprache, was es leicht macht - um ein Beispiel zu nennen -, das Körper-PsycheGrenzkonzept des Triebs zu übersetzen. Auch das gemeinsame Erbe von griechischlateinischen Ausdrücken für die Beschreibung von Krankheiten, die bereits vor mehr als 1000 Jahren von Ärzten und Philosophen wie Al-Rhazi und Ibn Sina ins Arabische aufgenommen wurden, ebnet den Boden für die Übersetzbarkeit der analytischen Konzepte (ebd., S. 4). Auch sind neue Begriffe für Ausdrücke, die durch Ableitungen von Namen geschaffen wurden, wie beispielsweise die Termini Masochismus oder Narzissmus, leicht zu bewerkstelligen. Überhaupt können im Arabischen einfach Neologismen geschaffen werden, weil arabische Begriffe allesamt auf eine Sprachwurzel zurückgehen, aus denen die verschiedenen Wortarten wie Verben, Nomen usw. ableitbar sind. Es gibt sogar die Möglichkeit, Wörter aneinanderzureihen, womitWörter wie «vorbewusst» oder «prägenital» übersetzt werden konnten (ebd., S. 4).

Doch dieserVorteil kann auch leicht zum Nachteil einer guten Übersetzung werden, geht es doch darum, so Ben Slama (ebd., S. 4), eine «construction inventive du comparable» zu schaffen und nicht darum, in der angestrengten Suche nach gleichwertigen Ausdrücken eine zweifelhafte Kopie zu bilden. Es brauche einen «Widerstand» bei der Übersetzungstätigkeit.

Die Schwierigkeiten der psychoanalytischen Verständigung innerhalb der arabischen Community sind teilweise auch auf eine historisch gewachsene Sprachverwirrung der arabischen Psychoanalyse zurückzuführen. Von Beginn an gab es keinen einheitlichen Sprachgebrauch, sondern die Übersetzenden psychoanalytischer Literatur versuchten stets, mit der für sie trefflichsten Wortwahl das eigene Übersetzungswerk zu vollenden. In den 1970er-Jahren, einer Zeit, in der Tarabishi an seinen Übersetzungen arbeitete, verschlimmerte sich die Lage: Es gab eine Polarisierung zwischen den tendenziell frühen ägyptischen und den 
libanesischen Übersetzungen: eine regionale Dichotomie, die sich bei der Wahl zentraler Begriffe wie beispielsweise der des Triebs, der Ambivalenz und sogar des Unbewussten zeigte (ebd., S. 7). Mit ein Grund dafür war die Tatsache, dass viele dieser um die Mitte des 20. Jahrhunderts in Ägypten individuell übersetzen Werke nur im Land selbst erschienen. Oft wurden diese nicht mehr neu aufgelegt. Zu ergänzen bleibt, dass es trotz dieses Problems der Polynomie auch ein grösseres Feld von relativ festen Begriffen in der arabischen Psychoanalyse gibt (ebd. , S. 8).

Angesichts der aktuellen Bemühungen in arabischen Glossaren, die Varianten psychoanalytischer Termini akribisch auszubreiten, schlägt Ben Slama vor, eine Institution zu gründen, die sich der Frage einer arabischen Konstruktion des psychoanalytischen Wissensgebäudes annehmen würde. Das Ziel wäre, ein vereinheitlichtes Vokabular für die arabofone Weltregion zu schaffen. Damit könnte das Fundament gelegt werden, um der Psychoanalyse in der arabischen Welt, jenseits von egozentrischen Passionen und der unantastbaren Autorität psychoanalytischer Überväter, zu besserer Position und eindeutigerem Profil zu verhelfen (ebd., S. 11).

\section{Die erste psychoanalytische Gesellschaft der arabischen Welt}

Die Konsolidierung der Psychoanalyse auf institutioneller Ebene hatte im Libanon - dem Land, das früher als die Schweiz und dessen Hauptstadt Beirut als das Paris des Nahen Ostens galt - am frühsten begonnen. Wichtig für die Entwicklung der Psychoanalyse waren Hochschulen, an denen Psychologie studiert werden konnte. Hierzu zählte namentlich die Université Saint-Joseph, eine christliche Privatuniversität, an der im Rahmen des Psychologiestudiums die Lehre der Psychoanalyse viel Gewicht hatte. Auch besassen die Dozierenden vielfach einen psychoanalytischen Hintergrund. Sie wurden zu Vorbildern für Studierende, sodass bei einigen der Wunsch aufkam, selbst eine psychoanalytische Laufbahn einzuschlagen. Um sich nicht nur über Bildungsaufenthalte in Frankreich zum bzw. zur PsychoanalytikerIn ausbilden zu können, aber auch um den vereinzelten im Libanon Praktizierenden ein Austausch- und Vernetzungsforum zu bieten, wurde die Société libanaise de psychanalyse (SLP) gegründet. 2010 schreibt eine libanesische Analytikerin rückblickend dazu: «Je m’incline devant leur courage qui leur a permis, contre vents et marées de constituter en 1980 la première société psychanalytique du Liban regroupant différents courants analytiques, et évitant ainsi les clivages européens» (Osseiran, 2010, S. 97).

Die drei Gründungsmitglieder, zwei Psychiater und ein Psychologe, hatten, wie die meisten anderen der dazumal an zwei Händen abzuzählenden 
PsychoanalytikerInnen, ihre Ausbildung in Frankreich gemacht. Einige davon wählten dazu die heute von der International Psychoanalytic Association (IPA) für Randgebiete unterstützte Form der Shuttle-Analyse. Diese wurde von den libanesischen AnalytikerInnen in Ausbildung jedoch gänzlich aus eigener Tasche finanziert: Eine knappe Woche pro Monat in Paris, während der man in gedrängter Weise die Lehranalyse absolvierte, den Rest des Monats im Libanon als PsychoanalytikerIn arbeitend. Wer nicht MedizinerIn war, hatte oft noch ein zweites berufliches Standbein, um den Lebensunterhalt bestreiten zu können.

Mit viel Stolz wird erzählt - so habe ich während meiner Feldforschung im Libanon erfahren -, dass die SLP die erste psychoanalytische Gesellschaft der arabischen Welt sei. Die Eigendefinition der SLP als Sammelbecken verschiedener Strömungen der Psychoanalyse ging Hand in Hand mit der Besonderheit des Libanon, 18 staatlich anerkannte Konfessionsgemeinschaften zu beherbergen. Dem religiösen Pluralismus zukünftiger Mitglieder sollte auch ein psychoanalytischer Pluralismus entsprechen. Ein wichtiger Grund für diesen angestrebten theoretischen Pluralismus war aber auch, damit die grosse Spaltung Frankreichs zwischen Freud- und Lacan-Gefolgsleuten zu umgehen. Diesen hehren Bemühungen, eine lokale Ausbildungsmöglichkeit anzubieten, machte der Bürgerkrieg jedoch bald einen Strich durch die Rechnung. Die mit Elan begonnen Seminare mussten mit der Zeit eingestellt werden.

\section{Psychoanalysieren im Bürgerkrieg}

Adnan Houbballah war einer der Gründer der SLP. Historisch betrachtet spielt er insofern eine Rolle, als er meines Wissens der erste muslimische Psychoanalytiker im Libanon war. Alle anderen frühen PsychoanalytikerInnen gehörten christlichen Konfessionen an oder waren französischer Nationalität.

Houbballah wurde 1937 in Beirut geboren. Für sein Medizinstudium begab er sich nach Marseille, wo er seine Assistenzarztzeit im Hôpital de Marseille absolvierte. Sein Zugang zur Psychoanalyse erfolgte über die Psychosomatik, ein Thema, in dem er auch dissertierte. Er gewann für diese Arbeit 1969 den Marseiller Prix des thèses. Houbballah entschloss sich zu einer ersten Tranche Psychoanalyse bei einem französischsprechenden Analytiker. Er setzte diese in Paris beim aus Ägypten stammenden Moustapha Safouan fort, um, ganz im Sinne der Psychoanalyse Lacans, zum signifiant arabe zu finden (Ayouch, 2002, S. 1). Dritter Psychoanalytiker, der für seinen Werdegang wichtig wurde, war schliesslich Jacques Lacan. Er war bei ihm über lange Jahre in Supervision. 
1973 kehrte Houbballah in den Libanon zurück und eröffnete seine Praxis im Westen Beiruts, in einem Quartier, das später vom Bürgerkrieg stark betroffen war. Es wurde eine Zeit des ständigen Pendelns zwischen Paris und Beirut. Erst ab 2004 wohnte er wieder ausschliesslich im Libanon (Chamoun \& Hoffman, o. A.). In Beirut wirkte er als Professor in klinischer Psychopathologie an der staatlichen libanesischen Universität und als Psychoanalytiker und Neuropsychiater (Ayouch, 2002, S. 1).

Houbballah war ein umtriebiger Psychoanalytiker. Neben seiner Tätigkeit für die SLP gründete er das Centre arabe de recherches psychanalytiques et psychopathologiques. Er setzte seine Supervision bei Lacan bis zu dessen Tod im Jahr 1981 fort. Angesichts der zunehmenden Zerstörungsgewalt und der Bedrohungen des Bürgerkriegs entschied sich Houbballah im Jahr 1987 zum vollständigen Exil nach Paris, wohin seine Familie bereits übersiedelt war (ebd.). Vor seiner Abreise war er oft von JournalistInnen aus der ganzen Welt aufgesucht worden, die von ihm, einem der letzten verbliebenen Psychiater und dem einzigen Psychoanalytiker in West-Beirut wissen wollten, wieso diesem Krieg, den alle verdammten, kein Ende zu bereiten war (Houbballah, 1996, S. 169).

Houbballah reflektierte während seiner psychoanalytischen Arbeit stets seine Position als Analytiker in einem arabo-muslimischen Umfeld. Er wirkte als Übersetzer psychoanalytischer Werke und verfasste auch eigene Arbeiten auf Arabisch wie beispielsweise eine Veröffentlichung zum Wandel der Psychoanalyse von Freud zu Lacan. Er schrieb ferner zwei analytische Werke aufgrund seiner Erfahrungen mit dem Bürgerkrieg (vgl. Houbballah, 1996, 1998). Nach vier Jahren in Paris kehrte er teilweise in seine Beiruter Praxis zurück. Wie Houbballah selbst schreibt, war ihm das Exil in dem Moment aufgezwungen worden, als ihn die Furcht, jederzeit attackiert zu werden, in der Arbeit behinderte-dies nach etlichen Jahren, in denen er den Kampfgefechten zum Trotz weitergearbeitet hatte, aus dem starken Gefühl heraus, dass dies von Nutzen für seine im Land verbliebenen PatientInnen sei (Houbballah, 1996, S. 196). Aufgrund der Bürgerkriegserfahrungen praktizierte Houbballah selten im klassischen Setting. In einem Kontext, in dem sich die PatientInnen jederzeit der Gnade des Schicksals ausgeliefert wussten und oft wagemutig in ihre Analysesitzungen eilten, hielten sie die liegende Position, in der sie vermehrt der Gewalt ihrer Vorstellungen ausgeliefert waren, nicht aus. Für Houbballah war klar, dass er in der Kriegssituation als Psychoanalytiker das Reelle, das jederzeit durch das Fenster hereinplatzen konnte, nicht ignorieren durfte. Vor diesem Hintergrund entstand auch seine Regel, die Tür zu seiner Praxis jederzeit offen stehen zu haben, offen für alle, die kommen wollten (Ayouch, 2002, S. 7). 
Die Angst vor den verschiedenen kämpfenden Gruppierungen führte indessen nicht allein zum Exil. Im Kern war es bei Houbballah das persönliche Konfrontiertsein mit dem Zerfall der zivilen Gesellschaft. Eines Morgens fand Houbballah im Aufzug des Hochhauses zu seiner Praxis folgende Inschrift vor: «Si le docteur Houbballah ne me soigne pas, je le tue» (Houbballah, 1996, S. 195). Houbballah begann sich zu fragen, wer diese Botschaft verfasst haben könnte. Es gelang ihm nicht, die Patientenliste auf einen kleinen Kreis von Infragekommenden zu reduzieren. Dies wurde für ihn zum Moment, in dem die Furcht vor äusserer Umgebungsgewalt nicht mehr von der subjektiven Angst zu unterscheiden war: ein Terror, der zum Verlust des freien und kreativen Denkens führte. Die Voraussetzung für sein psychoanalytisches Arbeiten war damit nicht mehr gegeben (Houbballah, 1996, S. 196).

Anzufügen ist, dass Houbballah aufgrund seiner Gewalt- und Kriegserfahrungen in seiner Pariser Zeit ein gesuchter Therapeut für PatientInnen wurde, die Opfer urbaner Gewalt geworden waren. Des Öfteren begleitete er auch humanitäre Organisationen bei ihren Hilfsaktionen für Kriegsopfer (Ayouch, 2002, S. 7). Er war einer der grossen Brückenbauer zwischen der westlichen Psychoanalyse und dem Nahen Osten und machte sich lebenslang für die Arabisierung der Psychoanalyse stark. Im Gegensatz zu vielen arabischen KollegInnen wählte er den Westen immer nur zeitlich begrenzt als seine zweite Heimat. 2009 starb Houbballah.

\section{Neue psychoanalytische Institutionen im Libanon, in Marokko und Tunesien}

Trotz des Wiederbelebens der Aktivitäten der SLP nach Beendigung des Bürgerkriegs zeichnete sich ab der Jahrtausendwende eine erneute Krise ab. Sie führte zu Austritten und zur Neugründung zweier weiterer psychoanalytischer Einrichtungen. Zwischenzeitlich gehörten einige libanesische AnalytikerInnen nur noch einer französischen psychoanalytischen Gesellschaft an. Zusätzlich traten aber auch arabische KollegInnen aus Frankreich der SLP zur Verstärkung bei.

Parallel zu diesen libanesischen Entwicklungen entstand im Jahr 2001 in Marokko die zweite psychoanalytische Gesellschaft der arabischen Welt, die Société psychanalytique marocaine (SPM). Auch diese versteht sich als Gremium aller an der Psychoanalyse interessierten, im Bereich der psychischen Gesundheit wirkenden Professionellen (Bennani, 2003). Institutioneller Vorläufer der SPM war die ab 1985 während vier Jahren aktive Gruppierung Le texte Freudien, ein Zirkel von Intellektuellen, künstlerisch und psychoanalytisch Tätigen, die an einer gemein- 
samen Reflexion über die Psychoanalyse interessiert waren und dazu Konferenzen organisierten (Bennani, 2005, S. 119).

Das formulierte Ziel der SPM war, die psychoanalytische Ausbildung und Praxis in Marokko selbst zu institutionalisieren sowie auch einen Garanten für die Einhaltung berufsethischer Aspekte darzustellen. Als dritte Variante der psychoanalytischen Ausbildung ersann die Gesellschaft neben dem Modell der individuellen, mit einem Auslandaufenthalt verbundenen Ausbildungen und dem der Shuttle-Analysen eine dritte Variante: das Einfliegen von europäischen Analytikern, die eine Woche im Monat in Marokko arbeiteten und bei denen in dieser Zeit die AusbildungskandidatInnen eine verdichtete Lehranalyse machen konnten.

Wie im Libanon bot auch in Tunesien eine Universität im Hintergrund das notwendige strukturelle Rückgrat für die Formierung einer psychoanalytischen Gesellschaft. Mit dem arabischen Frühling schossen hier viele neue Verbände und Gruppierungen aus dem Boden und erhielten staatliche Anerkennung. Darunter war auch die Association Tunisienne pour le Développement de la Psychanalyse (ATDP). Sie entstand 2011, angestossen durch Riadh Ben Rejeb, einem Psychologieprofessor der Faculté des sciences humaines et sociales de Tunis. Die ATDP ist Partnerin der Groupe méditerranéen der Pariser Gesellschaft für Psychoanalyse. Sie positioniert sich somit im Umfeld der mit der IPA affilierten Gesellschaften. Die Association de Formation à la Psychanalyse et d'Echanges Cliniques (AFPEC) wurde 2012 gegründet, u. a. von Fehti Benslama, einem ursprünglich tunesischen Analytiker, der 1972 nach Frankreich kam und sich mit Publikationen zum Verhältnis von Psychoanalyse und Islam einen Namen machte (Ben Smaïl, o.A.).

Fast zeitgleich formierten sich in Tunesien somit zwei analytische Gesellschaften. Der Trend, der den Libanon in den letzten zehn Jahren ereilte, lässt sich auch hier erkennen, nämlich die Genese von zwei sich voneinander abgrenzenden psychoanalytischen Feldern. Im Libanon bedeutete dies zum einen das Fortbestehen der SLP als pluralistische offene Gruppierung, unter deren Dach PsychoanalytikerInnen verschiedener Couleur ihren Platz haben; zum anderen die Rückbesinnung auf die Affiliationslinie der IPA. Dazu zählt die 2009 gegründete Association Libanaise pour le Développement de la Psychanalyse (ALDeP). Sie wurde 2010 als study group der IPA anerkannt und hat heute neun Mitglieder. Die GründerInnen waren mehrheitlich zuvor Mitglieder der SLP gewesen. Von der Grösse her gibt es nun im Libanon zwei gleich «kleine» Gesellschaften. Auch die SLP zählt aktuell neun Mitglieder (vgl. http:// www.slp-web.org). Die ALDeP selbst erklärt ihre Gründung damit, dass sie nicht riskieren wollte, isoliert dazustehen. 
Sie fürchtete, von der internationalen psychoanalytischen Bewegung abgekoppelt zu werden (vgl. http://www.aldep.org/presentation.php).

Neben der SLP und der ALDeP entstand im Libanon zudem die Ecole libanaise de psychanalyse und psychothérapie. Die Begründung zur Errichtung dieser Einrichtung lautet etwas anders: Der Initiator rechtfertigt sie mit der Varietät von an die 700 heute weltweit existierenden Psychotherapierichtungen. Diese würden der Psychoanalyse nolens volens als Konkurrenz erwachsen. Durch die Rigidität der psychoanalytischen Gesellschaften alter Schule - deren analytische Technik sich in eine Form der «Orthopraxie» transformiert habe - sei diese entmenschlicht worden (Azouri, o.A.). Ein Teil der Klientelen sei aus diesem Grund aus den psychoanalytischen Praxen geflohen und hätte sich in wohlwollenderen Psychotherapiepraxen niedergelassen. Durch den Schulterschluss mit einer Klinik, die als Praktikastätte für den psychoanalytischen Nachwuchs mitwirkt, ist geplant, eine solide, Praxis und Theorie verbindende Ausbildungsmöglichkeit zu schaffen. Es bleibt abzuwarten, inwieweit dieser von einer Einzelperson vollbrachte Kraftakt zu einer lebendigen Ausbildungseinrichtung anwachsen kann.

Auch Marokko, das nach dem Libanon als das arabische Land gilt, welches in der Etablierung der Psychoanalyse am aktivsten ist, blieb eine Abspaltung nicht erspart. Im gleichen Jahr, in dem die libanesische study group der IPA ihren Betrieb aufnahm, gründeten fünf PsychoanalytikerInnen den Cercle Psychanalytique Marocain (CPM). Sie hatten die SPM bereits zwei Jahre zuvor (2007) verlassen. Stein des Anstosses war die Vorgabe, sich bei einem bzw. einer der eingeflogenen AnalytikerInnen in Lehranalyse zu begeben. Innerhalb des neuen CPM ist es wieder möglich, in freier Wahl die eigene Lehranalyse zu gestalten (Bennani, 2010, S. 17).

\section{Auseinandersetzungen mit der Psychoanalyse in Ägypten}

Meine Einblicke in den Prozess der Institutionalisierung der Psychoanalyse im Machrek und im Maghreb habe ich bis hierhin aus einer modernisierungstheoretischen Perspektive verfasst. Diese baut implizit auf derVorstellung, dass es eine modellhafte Version der Psychoanalyse gibt, die sich über den Globus ausbreitet, wobei die arabische Welt diesen Verbreitungsbestrebungen widerstrebend oder unempfänglich gegenübersteht.

Die Frage des Fussfassens der Psychoanalyse in der arabischen Welt ist in vielen wissenschaftlichen Debatten erörtert und mit Verweis auf die Spezifika der islamischen Religion und Kultur beantwortet worden. Unter Einfluss der postcolonial studies ist diese Frage in letzter Zeit neu belebt worden. Leitgedanke des Diskurses ist: “[D]ifference is alreday differed, and therefore cannot be thought of 
as a concept having an original and a copy - neither West nor East holds the position of the original", wie Jalan formuliert (1997). Ihren Gedanken weiterspinnend bestehen weltweit somit verschiedene Formen der Psychoanalyse, die lokal geprägt und in Machtstrukturen eingebunden sind und die sich gegenseitig beeinflussen. Die Unterschiede zu der modernisierungstheoretischen Sichtweise derVerbreitung und Entwicklung der Psychoanalyse sind subtil. Sie zeigen sich jedoch in anders formulierten Fragen und Bezugspunkten, in der Definition der Akteurinnen und Akteure und in der Bewertung der gewählten Bezugspunkte.

Als Folge dieser Auseinandersetzung mit den postcolonial studies werden nun neue Facetten der arabischen Psychoanalyse-Entwicklung ins Zentrum gerückt. Es entstanden Studien, die jenseits von Pionier-, Expansions- und Normalisierungsphase arabische wie islamische ProtagonistInnen und ihre psychoanalytischen Fussabdrücke in der arabischen Welt sichtbar machen. Es handelt sich um Beiträge, die den Blick auf individuelle Aktivitäten legen und diese in einem grösseren Zusammenhang zu interpretieren versuchen: im Verhältnis von Westen und arabischerWelt wie auch im Verhältnis von früheren Kolonialländern und ihren ehemaligen Kolonialmächten. Gerade für Ägypten findet aus dieser Perspektive ein neues Aufrollen der psychoanalytischen Geschichtsschreibung statt.

Diese Geschichtsschreibung begnügt sich nicht mit der Wehklage, dass es zwischen den 1930er- bis 1960er-Jahren eine prosperierende psychoanalytische Bewegung gegeben hätte, die aufgrund der zunehmenden politischen Repression zerschlagen wurde. El Chakry (2014) zeigt eine andere Lesart dieser frühen Phase der Psychoanalyse in Ägypten auf. Sie arbeitet heraus, dass es sich dabei um eine Phase vielfältiger Interaktionen, der Hybridisierung der psychoanalytischen Theorie wie auch der eigenständigen Wissensproduktion zwischen Ägypten und Europa gehandelt habe: Ägyptische Intellektuelle aus Psychologie, islamischer Philosophie und den Rechtswissenschaften untersuchten ab den 1930er-Jahren Schlüsselkonzepte aus Psychoanalyse und Psychologie. Sie erörterten psychoanalytische Modelle und setzten diese mit klassischen islamischen Konzepten in Verbindung. Dabei glichen sie einzelne Theoriebausteine miteinander ab und schufen neue “blended”-Ansätze zu Fragen von Persönlichkeit und Individualität. In ihren Schriften betonten sie die Einheit des Selbst und machten gleichzeitig auf die Heterogenität des europäischen psychoanalytischen Denkens aufmerksam. Diese ägyptische Aneignungsleistung der Psychoanalyse und Schaffung eines hybriden psychologischen Wissensgebäudes dauerte bis in die späten 1950er-Jahre. El Chakry setzt sich damit von der Annahme, dass zwischen Islam und Psychoanalyse eine gegenseitige Ignoranz besteht, deutlich ab. Zentrale Figuren dieser ägypti- 
schen Auseinandersetzung mit der Psychoanalyse waren Yusuf Murad und Mustafa Radwan Ziywar, die beide in Europa studiert hatten (Ben Slama, 2010).

Kritischer beurteilt Ben Slama (ebd.) diese Zeit der Auseinandersetzung mit der Psychoanalyse. Sie bewertet sie weniger als gelungene hybride Wissensproduktion. Für sie ist die Psychoanalyse bis heute nicht in Ägypten angekommen, sondern wurde immer als Teil der Psychologie aufgefasst und im Kontext der Psychologie simplifiziert und ihrer Besonderheiten beraubt. Für Ben Slama bleibt die Frage offen, wie dieser eklektische Umgang mit der Psychoanalyse zu bewerten ist. Einerseits könnte es sich dabei um ein Zugeständnis an das der Psychoanalyse feindlich gesonnene Umfeld handeln. Andererseits wäre eine unbewusste Ambivalenz der ProtagonistInnen gegenüber der Psychoanalyse möglich, da sie in einem islamischen Land leben, dessen Bevölkerungsmehrheit sich als sehr religiös erklärt (ebd., S. 92). Diese Ambivalenz zeigt sich beispielsweise bei zwei Psychoanalytikern, die in den 1970er-Jahren, nach der ägyptischen Kriegsniederlage von 1967, die psychoanalytische Literatur daraufhin überprüften, ob sich der jüdische Feind «Israel» darin finden lässt. Diese Prüfung führte zur Publikation von Artikeln wie etwa «Freud entre la science et le sionisme» und bewirkte bei den beiden Analytikern schliesslich eine Ablehnung der Psychoanalyse (ebd., S. 87).

Die historisch frühe Phase der Psychoanalyse in Ägypten erlaubt drei Lesarten: erstens als Blütezeit einer neuen Institution und deren Zerfall aufgrund der politischen Verfolgung von PsychoanalytikerInnen; zweitens als produktive Phase der Formierung eines neuen, auf der Psychoanalyse fussenden, aber auch über diese hinausgehenden hybriden Wissens; oder drittens als Periode einer ambivalenten Begegnung mit einer neuen Disziplin, die der Psychologie untergeordnet wurde.

Den «quelques oiseaux rares» (Ben Slama, 2010, S. 85), die aktuell als AnalytikerInnen in Ägypten arbeiten, bleibt in diesem Sinne die Erkenntnis, dass das Ausüben der Psychoanalyse im Umfeld der arabisch-islamischen Kultur zwar erschwert ist, aber nicht verhindert wird. Eine prinzipielle Inkompatibilität findet sich in der Praxis nicht, und die Toleranz der islamischen Kultur gegenüber der Psychoanalyse sollte nicht unterschätzt werden. 


\section{Literatur}

Ansary, Tamim. (2010). Die unbekannte Mitte der Welt. Globalgeschichte aus islamischer Sicht. Frankfurt a. M.: Campus.

Ayouch, Thamy. (2002). Adnan Houbballah: de l'exil d'un psychanalyste à une psychanalyse de l'exil. Cairn.info, (Topique. Revue Freudienne, 3, 80, 81-88). http://www.cairn.info/revue-topique-2002-3-page-81.htm (abgerufen am 3. April 2015).

Azouri, Chawki \& Roudinesco, Elisabeth (Hrsg.). (2005). La psychanalyse dans le monde arabe et islamique. Beyrouth: Presses de l'USJ.

Azouri, Chawki. (o.A.). Ecole libanaise de psychanalyse et psychothérapie. http:// mlh.com.lb/departments/psychiatry/ecole-libanaise-de-psychanalyse-etde-psychotherapie/ (abgerufen am 3. April 2015).

Ben Slama, Raja. (2015). L'arbre qui révèle la forêt. Traductions arabes de la terminologie freudienne. Transeuropéennes. Revue international de pensée critique, 1-11. http://www.transeuropeennes.eu/en/articles/voir_pdf/ 106 (abgerufen am 3. April 2015).

Ben Smaïl, Nedra. (o.A.). Psychanalyse en Tunisie: actualités et perspectives. https:// sites.google.com/site/olivierdouvilleofficiel/articles/psychanalyse-entunisie-actualites-et-perspectives (abgerufen am 3. April 2015).

Bennani, Jalil. (2010). Psychanalyser au Maroc. In Sophie Mijolla-Mellor (Hrsg.), La psychanalyse au maghreb et au machrek. Topique. Revue Freudienne, 110 (S. 7-22). Le Bouscat cedex: L’Esprit du Temps.

Bennani, Jalil. (2005). Comment réinventer la psychanalyse au Maroc? In Chawki Azouri, Elisabeth Roudinesco (Hrsg.), La psychanalyse dans le monde arabe et islamique (S. 115-134). Beyrouth: Presses de l'USJ.

Bennani, Jalil. (2003). La psychanalyse au Maroc. Historique et état des lieux. Psychanalyse Appliquée. Propositions de psychothérapie et publication de textes divers sur des sujets liés à la psychanalyse. http://psyapp.over-blog. com/article-31054065.html (abgerufen am 3. April 2015).

Bruno, Pierre. (2012). Présentation. In Rafah Nached (2012). Psychanalyse en Syrie (S. 9-16). Toulouse: Editions érès.

Chamoun, Mounir \& Hoffmann, Christian. (o. A.). Adnan Houbballah (1937-2009). Oedipe. http://www.oedipe.org/fr/adds/pa?mode=view\&id=107 (abgerufen am 3. April 2015).

EDK, (Hrsg.). (2004). La psychanalyse et le monde arabe. La célibataire. Revue de psychanalyse, 8. 
El Shakry, Omnia. (2014). The Arabic Freud. The unconscious and the modern subject. Modern Intellectual History, 11, 1, 89-118. https:/ /www.academia. edu/6247448/The_Arabic_Freud_The_Unconscious_and_the_Modern_ Subject (abgerufen am 3. April 2015).

Hartmann, Katrin. (2007). Die Psychoanalyse im Libanon. Berlin: Verlag Hans Schiler.

Houbballah, Adnan. (1998). Destin du traumatisme. Comment faire son deuil. Paris: Hachette Littératures.

Houbballah, Adnan. (1996). Le virus de la violence. La guerre civile est en chacun de nous. Paris: Albin Michel.

Jalan, Rajika. (1997). An Asian Orientalism? Libas and the Textures of Postcolonialism. In Alan Scott (Hrsg.). The Limits of Globalization. Cases and Arguments (S. 90-115). New York: Routledge.

Mijolla-Mellor, Sophie (Hrsg.) (2010). La psychanalyse au maghreb et au machrek. Topique. Revue Freudienne, 110. Le Bouscat cedex: L'Esprit du Temps.

Nached, Rafah. (2012). Psychanalyse en Syrie. Toulouse: Editions érès.

Nached, Rafah. (2010). Histoire de la psychanalyse au Syrie. In Sophie MijollaMellor (Hrsg.), La psychanalyse au maghreb et au machrek. Topique. Revue Freudienne, 110 (S. 117-127). Le Bouscat cedex: L'Esprit du Temps.

Osseiran, Mouzayan. (2010). De quelques difficultés de la pratique psychanalytique au Liban. In Sophie Mijolla-Mellor (Hrsg.), La psychanalyse au maghreb et au machrek. Topique. Revue Freudienne, 110 (S. 97-104). Le Bouscat cedex: L'Esprit du Temps.

Savigneau, Josyane. (2011). Rafah Nached et les prisons de la peur. http://www. cifpr.fr/Bulletin-de-la-societe,290 (abgerufen am 3. April 2015).

Schülein, Johann August. (2007). Optimistischer Pessimismus. Über Freuds Gesellschaftsbild. Göttingen: Vandenhoeck \& Ruprecht.

Zamyn. (o.A.). Interview mit Moustapha Safouan. http://www.zamyn.org/interviews/maccabe-safouan/interview.html (abgerufen am 3. April 2015).

Zoueïn, Josette \& De Rochegonde, Thierry. (2004). Rencontre avec un traducteur en arabe, Georges Tarabichi. Cairn.Info (Che vuoi?, 1, 21, 93-99). http:/ / www. cairn.info/revue-che-vuoi-2004-1-p-93.htm (abgerufen am 3. April 2015).

\section{Anmerkung}

1 Exemplarisch dafür: «Orient a été décentré par l'Occident: avec le terrorisme nous sommes malheureusement de nouveau au centre de l'Occident, et vous comprenez à quel point ceci nourrit le narcissisme» (zit. nach Zoueïn, de Rochegonde, 2004, S. 4). 